\title{
Dimensionamento estrutural de estradas de mina a céu aberto
}

\author{
Haul roads structural design in open pit mines
}

Lilian Masetti Lobo Soares de Sousa

Engenheira de Minas,

Mestre pelo Departamento de

Pós-Graduação em Engenharia Mineral,

Escola de Minas,

Universidade Federal de Ouro Preto.

Imasetti@gmail.com

\section{Waldyr Lopes de Oliveira Filho}

Doutor, Professor do Departamento de

Pós-Graduação em Engenharia Mineral,

Escola de Minas,

Universidade Federal de Ouro Preto.

waldyr@em.ufop.br

\section{Hernani Mota de Lima}

Doutor, Professor do Departamento de Pós-Graduação em Engenharia Mineral, Escola de Minas,

Universidade Federal de Ouro Preto.

hernani.lima@ufop.br

\section{Resumo}

Uma estrada de mina foi dimensionada com materiais típicos ocorrentes em mineração de minério de ferro no Brasil, utilizando-se os métodos empíricos CBR e DNER. Através do programa de dimensionamento estrutural mecanístico-empírico ELSYM5, compararam-se os resultados obtidos pelos métodos anteriores e propôs-se a otimização do dimensionamento feito pelo método DNER. Os resultados mostram que a espessura total do pavimento calculada pelos dois métodos é, basicamente, a mesma, sendo que a espessura da camada de base pelo método DNER é superior à obtida pelo CBR, enquanto o oposto ocorre com a sub-base. Ambos os dimensionamentos mostram, através da aplicação do software ELSYM5, que não são os mais apropriados, visto que a deformação de algumas camadas, principalmente no caso CBR, está acima do limite aceitável de $2000 \mu \varepsilon$. Através da variação da espessura das camadas, obteve-se um dimensionamento adequado, que levou à redução da espessura da camada total do pavimento de 1,97 m para $1,40 \mathrm{~m}$. A redução da deformação das camadas, em que se variou o módulo de elasticidade, demonstra a importância da realização de ensaios de caracterização física dos materiais.

Palavras-chave: Estrada de mina, dimensionamento estrutural, métodos empíricos CBR e DNER, método mecanístico-empírico.

\begin{abstract}
An unpaved mine haul road, based on CBR and DNER empirical methods, was designed using materials which are typical in current Brazilian iron ore mining. By means of the mechanistic-empirical structural ELSYM5 software, the results obtained by previous methods were compared and an optimization of the DNER design was proposed. The results demonstrate that the total thickness of the pavement calculated by the two methods is basically the same and that the base layer thickness calculated by the DNER method is superior to the one obtained by the CBR method, whereas the opposite occurs in relation to the sub-base. Both designs show through the application of ELSYM5 software that they are not the most appropriate, since the strain of some layers, especially in the CBR case, is above the acceptable limit of 2000 $\mu \varepsilon$. Analyzing the variation of the layer's thickness, it was possible to obtain an adequate design, which led to the reduction of the total pavement layer thickness from $1.97 \mathrm{~m}$ to $1.40 \mathrm{~m}$. The reduction of the layer's strain in which the elastic modulus was submitted to variation, demonstrates the importance of performing tests for the physical characterization of materials.
\end{abstract}

Keywords: Mine haul road, structural design, CBR and DNER empirical methods, mechanistic-empirical methods. 


\section{Introdução}

Estradas mineiras são normalmente projetadas por profissionais de planejamento de lavra e executadas por equipes de operação ou infraestrutura de mina. Ao operacionalizar uma cava final, o objetivo primordial do engenheiro é projetar acessos que considerem o máximo aproveitamento de minério associado à menor remoção de estéril, assim como as menores distâncias de transporte das frentes de lavra aos locais de destino. Em geral, o estudo das estradas mineiras considera, basicamente, alguns parâmetros de projeto geométrico, tais como declividades de rampa, largura e raio de curvatura, mas não é voltado aos elementos constituintes dos projetos estrutural, funcional e de drenagem. Essa situação produz um desempenho das estradas de mina bastante questionável, em muitos casos, e representa, nos dias de hoje, certo descompasso com o alto nível já alcançado em termos das tecnologias de explotação, incluindo-se, no caso, os veículos de transporte (Oliveira Filho et al., 2010).

Dos elementos de projeto de uma estrada mineira, o projeto estrutural tem sido o menos utilizado, talvez por exigir uma formação e um conhecimento pouco disponíveis em nosso meio. O estudo teórico do projeto estrutural de dimensionamento de pavimentos é tratado na disciplina Mecânica dos Pavimentos, nos cursos de Engenharia Civil e de Engenharia de Minas, principalmente no primeiro. Nessa área, a literatura específica, voltada ao dimensionamento de estradas de mina, é bastante escassa. Por tal razão, somada a outras de ordem, principalmente, econômica e de segurança ocupacional, a adaptação e a aplicação de conceitos advindos da referida disciplina mostram-se, particularmente, úteis em projetos de estrada de mina.

$\mathrm{O}$ projeto estrutural, segundo Hugo (2005), objetiva o dimensionamento das camadas que devem compor o pavimento. A capacidade de suporte dessas camadas deve ser tal que resista aos carregamentos impostos à estrada durante a sua vida útil de projeto, minimizando, na medida do possível, a necessidade de manutenções excessivas.

Thompson (1996) enfatiza que o projeto estrutural é um processo de composição do arranjo mais econômico das camadas do pavimento (considerando sua espessura e tipos de material disponível), que seja compatível com o material in situ e com o volume de tráfego previsto para a vida útil das estradas.

O pavimento dimensionado é formado por camadas de revestimento, base e sub-base, que são projetadas e construídas acima do terreno normalmente constituído pelo subleito in situ. Enquanto o revestimento tem propriedades funcionais, as outras camadas exercem papel estrutural.

Os objetivos desse artigo são: i) Apresentar o dimensionamento das camadas de base e sub-base de um pavimento de estrada em mineração típica de minério de ferro a céu aberto através dos métodos empíricos que usam o Índice de Suporte Califórnia ou CBR (California Bearing Ratio) em duas propostas: uma delas baseada na carga de roda, mais comum em aplicações na mineração, e a outra adaptada do Departamento Nacional de Estradas de Rodagem (DNER), atual Departamento Nacional de Infraestrutura de Transportes (DNIT), mais comum em estradas rodoviárias; ii) Otimizar o dimensionamento obtido pelo método do DNER.

O programa de análise estrutural mecanístico-empírico ELSYM5 foi usado para comparar os resultados obtidos pelos dois métodos empíricos e para suportar a validação da otimização do método do DNER.

\section{Materiais e métodos}

\section{Materiais de construção e pontos de análise}

Os materiais usados, no dimensionamento das camadas do pavimento, foram selecionados a partir de solos e de rochas tipicamente encontrados na explotação de minas de ferro e/ou produtos resultantes do processamento mineral desses materiais no Brasil. A Tabela 1 mostra a proposição de utilização dos materiais e suas principais características.

Os CBRs do revestimento, base e sub-base, foram obtidos em laboratório, pelo ensaio Índice de Suporte California - NBR 9895/87, ou, no campo, via utilização do equipamento DCP (Dynamic

\begin{tabular}{c|c|c|c}
\hline Material & Utilização no dimensionamento & CBR (\%) & E (MPa) \\
\hline Rejeito de jigue & Revestimento & 34 & 150 \\
\hline Itabirito compacto & Base & 80 & 200 \\
\hline Canga laterítica & Sub-base & 26 & 120 \\
\hline Solo argiloso & Subleito & 3 (estimado) & 65 \\
\hline
\end{tabular}

\section{Dimensionamento de pavimentos}

As metodologias dos métodos empíricos utilizados no dimensionamento dos pavimentos, nessa pesquisa, usam o Índice de Suporte Califórnia ou CBR
Cone Penetrometer). Para o subleito in situ, foi considerado solo argiloso com CBR próximo de 3\%. A Tabela 1 inclui, também, os valores típicos médios dos módulos de elasticidade dos materiais (E), atribuídos a partir de Thompson e Visser (1999).

Tabela 1

Propriedades dos materiais.

\section{Método carga de roda (CBR)}

O dimensionamento das camadas foi feito tomando-se, como referência, o ábaco apresentado por Tan- nant e Regensburg (2001), para carga de roda, que tem, por base, a aplicação da teoria da elasticidade. Nesse
(California Bearing Ratio), em duas propostas. ábaco, são traçadas curvas contendo os valores CBR do material ensaiado contra a espessura da camada para 
vários carregamentos de roda. Determina-se a espessura total do pavimen-

\section{Método do DNER}

Esse método utiliza o número equivalente de operações de eixo-padrão (número N), fator esse que leva em conta, não só o carregamento (ou seu equivalente), mas, também, sua repetição (frequência), no período do projeto e o clima. O número $\mathrm{N}$ tem, como um dos multiplicadores, o fator de equivalência de operação (FEO), o qual se baseia no eixo simples padrão roda dupla de $80 \mathrm{kN}$ (8,2 tf) (DNIT, 2006).

Ferreira (2007) mostra que o método apresenta dificuldades para a sua

\section{Análise de tensões e deformações}

Para a avaliação do comportamento estrutural dos dimensionamentos feitos pelos métodos CBR e DNER, foi utilizado o programa ELSYM5. Ele calcula as componentes de tensão, deformação e deflexão em um sistema cartesiano tridimensional semi-infinito considera-

\section{Figura 1}

Pontos de análise de tensões e deformações (modificado de CAT, 2010).

\section{Resultados e discussão}

O estudo comparativo sobre os resultados de dimensionamento do pavimento, segundo os dois métodos CBR e DNER, está apresentado a seguir. Além desse comparativo, foram feitas dez simulações, visando a otimizar o dimensionamento das camadas obtido pelo

\section{Dimensionamento do pavimento}

Com base nos valores de entrada de dados discutidos e apresentados no item 2, a Tabela 2 apresenta o resultado dos dimensionamentos feito pelos métodos CBR (carga de roda) e DNER.

A espessura total de cobertura obtida pelos dois métodos é, praticamente, to, que deverá ser construído sobre o subleito. Também são determinadas as espessuras das camadas de subbase e base. aplicação em estradas de mina. A carga, por eixo, aplicada ao pavimento por caminhões fora-de-estrada excede imensamente o valor máximo do DNER, correspondente a $196 \mathrm{kN}$ (20 tf).De fato, a carga por eixo traseiro dos caminhões em estudo é 1638 kN (167 tf) (Caterpillar 785D) e $2520 \mathrm{kN}$ (257 tf)(Caterpillar 793D), sendo tais valores, aproximadamente, 8 e 13 vezes maiores, respectivamente, do que o valor máximo de carga por eixo previsto por esse método.

Dadas essas restrições, foi necessário extrapolar o FEO para as altas aplicações de carga por eixo, devido aos caminhões desse porte. A partir da equação de melhor ajuste alcançada por regressão potencial, foram obtidos os valores de FEO correspondentes às cargas por eixo de interesse.

Utilizou-se o ábaco do DNIT para fazer o dimensionamento do pavimento (DNIT, 2006). Foi necessário extrapolar as curvas para $\mathrm{N}$ igual a $1 \times 10^{14}$, valor este usado nos dimensionamentos, sendo esse valor mais alto do que o máximo contemplado pelo ábaco $\left(1 \times 10^{9}\right)$. do elástico. Admite-se, também, que as cargas aplicadas são, uniformemente, distribuídas sobre uma área circular na superfície do sistema (United States Department of Transportation, 1986).

A Figura 1 mostra os cinco pontos de análise das tensões, deformações e deflexões ao longo do eixo traseiro do caminhão Caterpillar 793D de $2520 \mathrm{kN}$ (257tf). Esses valores são usados na avaliação dos dimensionamentos através do programa ELSYM5, conforme será visto na sequência.

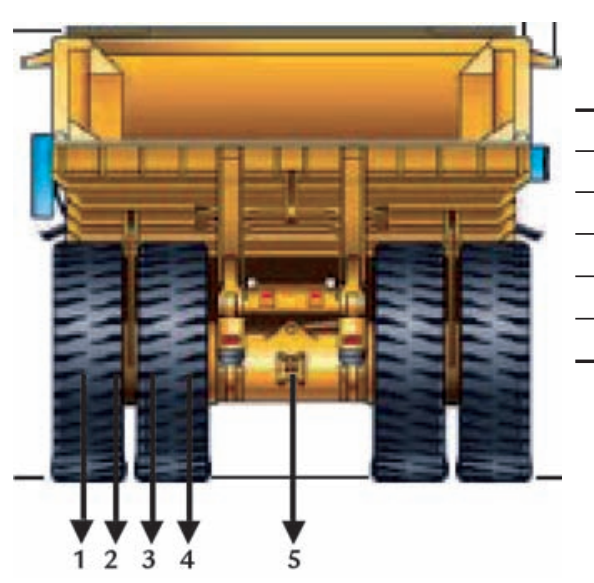

\begin{tabular}{c|c}
\hline Ponto & Distância (m) \\
\hline 1 & 0,00 \\
\hline 2 & 0,66 \\
\hline 3 & 1,32 \\
\hline 4 & 1,98 \\
\hline 5 & 3,80 \\
\hline
\end{tabular}

método do DNER. Em seis simulações, denominadas C1 a C6, foram alteradas espessuras de camadas, mantendo-se os valores dos módulos de elasticidade (E) (Thompson e Visser, 1999). Nas outras quatro simulações (C7 a C10), os valores de $\mathrm{E}$ dos materiais foram alterados. Dos dez casos de análise, aqui são apresentados os mais expressivos, correspondendo a C6, C7, C8, C9 e C10. Salienta-se que não se objetivou otimizar o dimensionamento da camada de revestimento, que deve ser tratada em separado, em estudos de projeto funcional de estradas. a mesma. A maior diferença entre camadas ocorre na base, em que o dimensionamento pelo método do DNER determinou uma espessura duas vezes e meia maior que a prevista pelo método CBR.

A espessura total do pavimento dimensionado pelo método CBR é muito sensível ao CBR do subleito, para valores de até $15 \%$, aproximadamente. Considerando o CBR do subleito aumentado de 1 ponto percentual $(4 \%)$, a espessura total das camadas dimensionadas acima deste seria reduzida de $1,96 \mathrm{~m}$ para $1,66 \mathrm{~m}$.

Quanto menor a capacidade de su- 
porte do subleito, maior será a espessura necessária do pavimento acima dele, de forma a se reduzirem sobre o mesmo, as tensões e deformações resultantes da carga de roda aplicada em superfície. Daí a importância da determinação, via ensaios laboratoriais, do valor mais exato possível do CBR das camadas a serem dimensionadas e, em especial, do subleito in situ.

$\mathrm{O}$ método $\mathrm{CBR}$ não leva em consideração as propriedades do revestimento, visto que a sua espessura é calculada pela distância entre o topo da camada de base e a superfície do pavimento dimensionado.

Benevides (2000), apropriadamente, salienta que o método do DNER não privilegia camadas de sub-base com CBR superior a $20 \%$. Ainda que o CBR da sub-base seja maior do que esse valor, a espessura do pavimento necessária para protegê-la é calculada como se o valor máximo de CBR da sub-base fosse $20 \%$. A espessura da sub-base poderia ser inferior, caso o método levasse

\begin{tabular}{c|c|c|c|c}
\hline Método & Revestimento $(\mathbf{m})$ & Base $(\mathbf{m})$ & Sub-base $(\mathbf{m})$ & Espessura total da camada $(\mathbf{m})$ \\
\hline CBR & 0,20 & 0,25 & 1,51 & 1,96 \\
\hline DNER & 0,13 & 0,63 & 1,21 & 1,97 \\
\hline
\end{tabular}

\section{Análise comparativas das deformações e deflexões}

em conta o CBR efetivo. Lembra-se que seria importante checar o desempenho estrutural dessa alteração, em termos de deformações admissíveis. Ainda assim, vale ressaltar que, partindo-se do princípio que materiais menos nobres são usados nas camadas inferiores, a redução da espessura da base contra o aumento da espessura da sub-base traria uma diminuição no custo, associada à necessidade de utilização de menor quantidade de material nobre.

\section{Tabela 2}

Comparativo entre os

dimensionamentos estruturais CBR e DNER.
A Figura 2 apresenta o comparativo das análises de deformações e deflexões máximas do pavimento dimensionado, segundo os métodos CBR e DNER, avaliados pelo programa ELSYM5. Percebe-se que a deflexão vertical máxima ocorre no eixo de cada pneu e tem valores próximos de acordo com os dois dimensionamentos. Para as deformações, no entanto, as diferenças são bastante expressivas, quando se comparam os dois métodos. No caso do CBR, boa parte do pavimento apresenta deformações superiores aos $2000 \mu \varepsilon$, que, segundo estudos de Thompson e Visser (1999), estariam

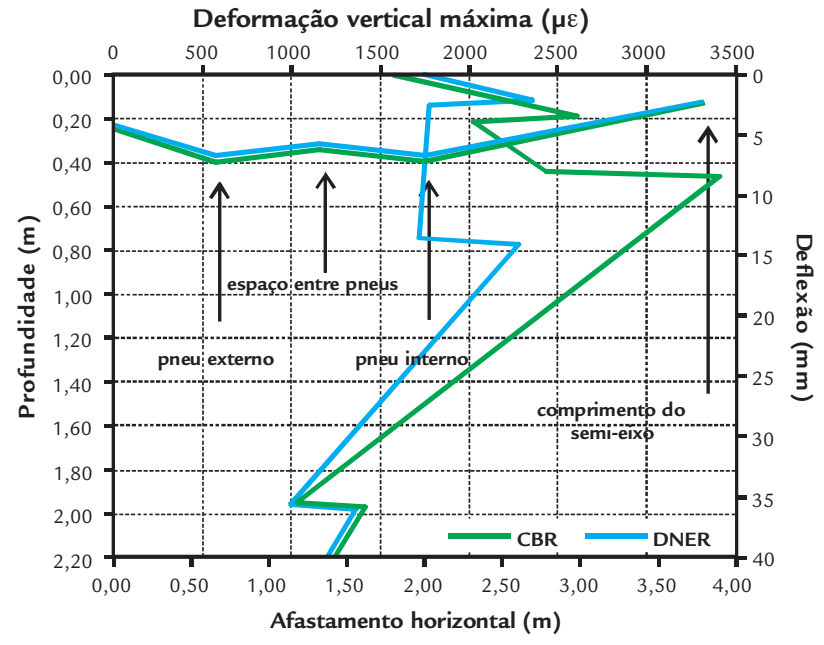

\section{Otimização da seção (DNER)}

Com a finalidade de diminuir a espessura total do pavimento e ao mesmo tempo respeitar o limite de deformação das camadas em $2000 \mu \varepsilon$, foram feitas simulações de otimização do pavimento. A Tabela 3 mostra a alteração na espessura das camadas de base e sub-base, levando ao resultado otimizado C6. A Figura 3 mostra o comparativo entre o dimensiona- mento original DNER e o caso C6. Notase que a espessura total do pavimento foi reduzida de $1,97 \mathrm{~m}$ para $1,40 \mathrm{~m}$.

A Figura 4 mostra isolinhas de deformação $(\mu \varepsilon)$ em valores absolutos de C6 interpoladas pelo programa Surfer ${ }^{\circledR}$.

As isolinhas de deformação de C6 mostram a influência da superposição dos bulbos de tensão gerados por mais de associados a um comportamento estrutural inadequado, fato não desejado, ao passo que apenas uma pequena espessura da sub-base encontra-se nessa situação, no caso do DNER. Já os efeitos no subleito são os mesmos como era de se esperar (mesma espessura total de cobertura).
Figura 2

Comparativo entre os

dimensionamentos estruturais CBR e DNER.
Tabela 3

Dimensionamento otimizado

(C6) comparado com o dimensionamento DNER.

\begin{tabular}{c|c|c|c|c}
\hline Método & Revestimento $(\mathbf{m})$ & Base $(\mathbf{m})$ & Sub-base $(\mathbf{m})$ & Espessura total da camada $(\mathbf{m})$ \\
\hline CBR & 0,13 & 0,63 & 1,21 & 1,97 \\
\hline DNER & 0,13 & 0,73 & 0,54 & 1,40 \\
\hline
\end{tabular}

um pneu ao longo do eixo do caminhão, efeito estudado por Kumar (2000). A partir de certa profundidade, a deformação localizada na abscissa entre pneus adjacentes supera a verificada nas abscissas sob os pneus, sendo isto especificamente notado no contato entre as camadas de sub-base e subleito. A curva de deformação $2025 \mu \varepsilon$, na Figura 4, indica tal efeito. 
Figura 3

Comparativo entre o dimensionamento original DNER e o caso 6.

Figura 4

Curvas de iso deformação de C6.

\section{Estudos sensitivos}

Os efeitos do uso de materiais alternativos ou variações construtivas (técnicas ou qualidades diferentes) podem ser avaliados por meio de mudanças nos parâmetros constitutivos dos materiais do pavimento. Nos casos C7 e C8, foram feitas alterações nos valores dos módulos de elasticidade E da sub-base e base de C6, respectivamente, sem alteração na espessura das camadas. Em C7, o valor de E da canga laterítica da sub-base de C6 foi alterado de $120 \mathrm{MPa}$ para $150 \mathrm{MPa}$, sendo este o valor médio entre todas as leituras obtidas por Saraiva (2006), atra-

\section{Conclusões}

$\mathrm{O}$ artigo explorou as possibilidades de um projeto estrutural de pavimento em estradas de mina, examinando duas propostas com base no ensaio CBR. Apesar de os dimensionamentos feitos pelos métodos CBR e DNER fornecerem, praticamente, a mesma espessura total, a análise tensão $\mathrm{x}$ deformação demonstrou que o método DNER produz pavi-
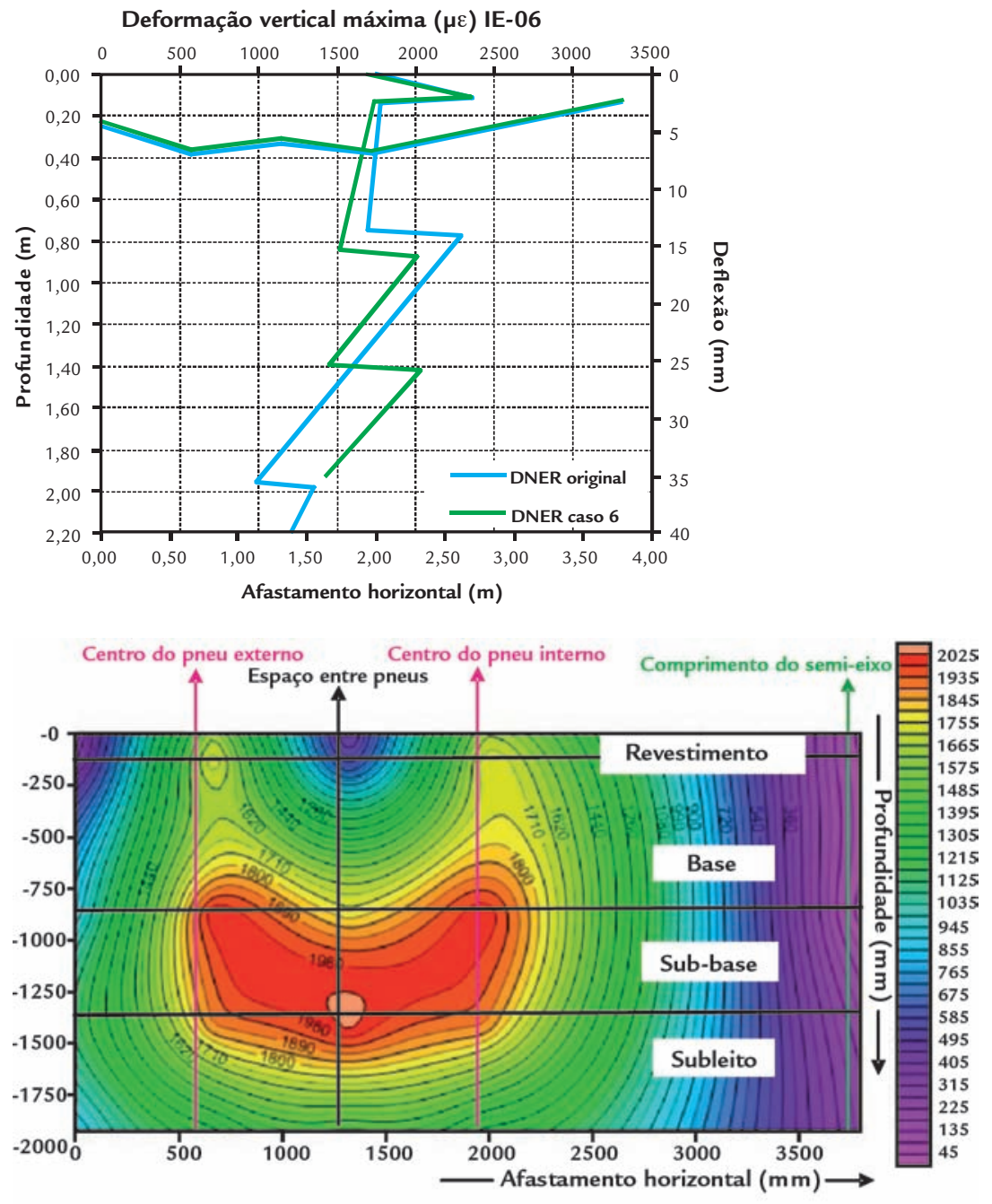

vés de ensaios de ressonância em amostra semelhante de argila. Em C8, o valor de E do itabirito da base de C6 foi alterado de $200 \mathrm{MPa}$ para $364 \mathrm{MPa}$, sendo esse valor assumido a partir de Saraiva (2006), através de ensaios de ressonância em amostra semelhante de cascalho de itabirito. A Figura 5 mostra os resultados dessas modificações, com uma redução expressiva das deformações.

Considerando-se a projeção e a construção de estradas sobre terrenos mais competentes, foram realizadas mais duas simulações. $\mathrm{O}$ valor de $\mathrm{E}$ do subleito de
C6 foi alterado de $65 \mathrm{MPa}$ para $120 \mathrm{MPa}$ (materiais com CBR entre 10\% e 14\%) e $135 \mathrm{MPa}$ (CBR acima de 25\%), casos C9 e C10, respectivamente (Figura 5). As alterações efetuadas provocaram redução acentuada no valor das deformações do subleito e da base. Esse fato aponta para a ideia de que poderia ser estudada uma otimização das seções dos casos C7, C8, C9 e C10, com possível redução na espessura total do pavimento. $\mathrm{O}$ comparativo da Figura 5 mostra, ainda, como terrenos mais competentes tem efeito considerável na redução das deflexões geradas. mentos mais bem dimensionados, isto por apresentar valores de deformação das camadas individualmente menores que os do CBR, e quase sempre dentro do limite tolerável de $2000 \mu \varepsilon$. Os estudos mostraram, também, que a seção do DNER pode ser otimizada com o uso de um programa de análise de tensões e deformações como o ELSYM5, fazendo um uso mais racional dos recursos materiais e de serviços de construção (menores coberturas e, portanto economia na implantação). Outro fato que chama atenção nas análises é que a seção do pavimento é bastante influenciada pelas condições dos terrenos de implantação e isso pode ser facilmente avaliado pelos métodos de dimensionamento. 


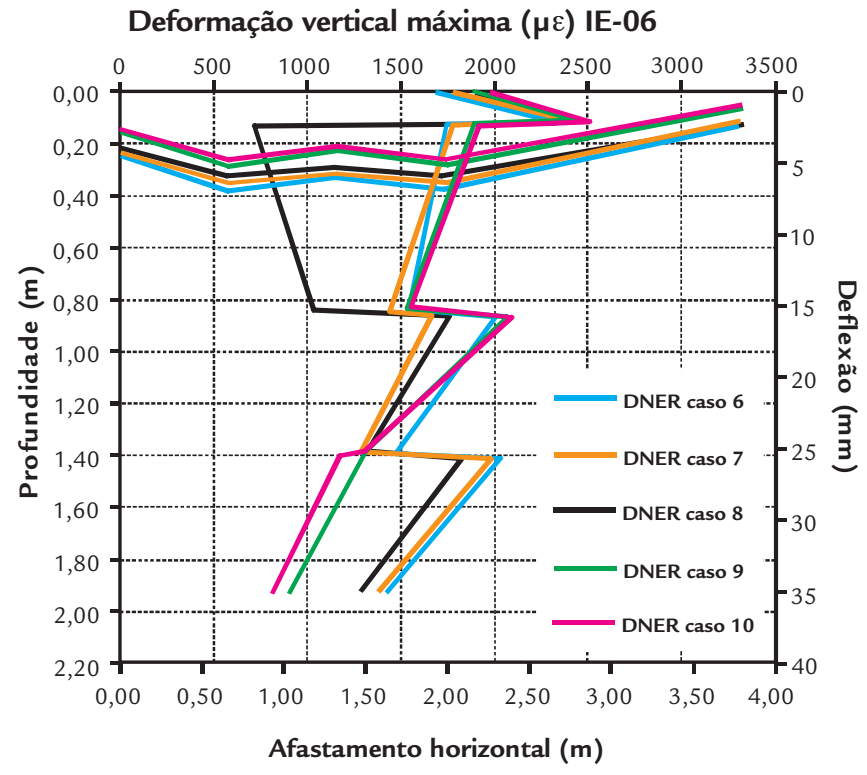

\section{Referências bibliográficas}

BENEVIDES, S. A. S. Análise comparativa dos métodos de dimensionamento de pavimentos asfálticos: empírico do DNER e da resiliência da COPPE/UFRJ em rodovias do estado do Ceará. Rio de Janeiro: Universidade Federal do Rio de Janeiro, 2000. 176 f. (Dissertação de Mestrado em Engenharia de Transportes).

CAT. 793D Mining Truck Manual. USA:2010. 32 p.

DNIT. Manual de pavimentação. (3 ed.). Rio de Janeiro, 2006. 274 p.

FERREIRA, R. M. Dimensionamento de um pavimento experimental para o tráfego de caminhões fora-de-estrada em planta de mina. Ouro Preto: Núcleo de Geotecnia, Escola de Minas da Universidade Federal de Ouro Preto, 2007. 277 f. (Dissertação de Mestrado em Geotecnia).

HUGO, D. Haul road defect identification and condition assessment using measured truck response. Department of Mechanical and Aeronautical Engineering, University of Pretoria, Pretoria, 2005. 109 f. (Dissertation of Master of Engineering).

KUMAR, V. Design and construction of haul roads using fly ash. Alberta: Department of Civil and Environmental Engineering, University of Alberta, 2000. $97 \mathrm{f}$. (Dissertation of Master of Science in Mining Engineering).

OLIVEIRA FILHO, W. L. de, FERNANDES, G., SOUSA, L. M. L. S. de, COSTA FILHO, W. D., RODRIGUES, C. A. F., VAN HAM, G. H. J. Relatório de Inventário de Estradas de Acesso de Mina. Convênio Vale UFOP, 2010. 12 p. (Relatório interno).

SARAIVA, S. L. C. Metodologia e análise experimental do comportamento geotécnico da estrutura de pavimentos rodoviários. Ouro Preto: Núcleo de Geotecnia, Escola de Minas da Universidade Federal de Ouro Preto, 2006. 123 f. (Dissertação de Mestrado em Geotecnia).

TANNANT, D. D., REGENSBURG, B. Guidelines for mine haul road design. School of Mining and Petroleum Engineering, Department of Civil and Environmental Engineering. Alberta: University of Canada, 2001. 108 p.

THOMPSON, R. J. The design and management of surface mine haul roads. Pretoria: Department of Civil Engineering, University of Pretoria, 1996. (Thesis of Doctor of Engineering)

THOMPSON, R. J. \& VISSER, A. T. Designing and managing unpaved opencast mine haul roads for optimum performance. In: SME ANNUAL MEETING, Anais... Denver: SME, 1999. Pre Print 99-90, 9 f.

UNITED STATES DEPARTMENT OF TRANSPORTATION. ELSYM5: Interactive Microcomputer Version. User's manual for IBM-PC and compatible microcomputer. Federal Highway Administration Report n FHWA -TS-87-206, 1986. $32 \mathrm{f}$.
Figura 5

Comparativo entre C6, C7, C8, C9 e C10.

Artigo recebido em 04 de agosto de 2011. Aprovado em 18 de outubro de 2011. 